\title{
Antibacterial Activity of Dopamine Incorporated Total Etch Adhesive System
}

\author{
${ }^{1}$ Adimulapu H Sandeep, ${ }^{2}$ Madhana Madhubala M, ${ }^{3}$ Sekar Mahalaxmi
}

\begin{abstract}
Aim: To evaluate the antibacterial efficacy of dopamine incorporated total-etch adhesive against Streptococcus mutans (S. mutans).
\end{abstract}

Materials and methods: $S$. mutans were cultured and inoculated in Müeller-Hinton agar plates. Round wells of around $6 \mathrm{~mm}$ diameter were created in the center of the agar plates. The experimental groups consisted of agents as follows: group I (DOPA 3\%), group II (DOPA 3\% + Bonding agent), group III (Bonding agent) and group IV (no material). For each group, 12 plates were used to evaluate the zone of inhibition using agar well diffusion method. All the experimental agents were added to respective wells in agar plates and incubated at $37^{\circ} \mathrm{C}$ for 24 hours. The diameter of a zone of inhibition around each well was recorded. Results were tabulated and analyzed statistically using the Kruskal-Wallis test. and Mann-Whitney U-test.

Results: Based on the mean diameters, group II showed the maximum zone of inhibition, and it exhibited statistically higher antibacterial activity than group III and group I $(p<0.01)$.

Conclusion: Within the limitations of this study, dopamine incorporated total-etch adhesive system exhibited significantly higher antibacterial activity than conventional adhesive system against S. mutans.

Clinical significance: Dopamine can be a promising antibacterial additive to dental adhesive systems to improve both biological seal and bond strength at the resin-dentin interface.

Keywords: Antibacterial, Dopamine, S. mutans, Total etch adhesive

How to cite this article: SandeepAH, Madhubala MM, Mahalaxmi S. Antibacterial Activity of Dopamine Incorporated Total Etch Adhesive System. J Oper Dent Endod 2018;3(2):53-56.

Source of support: Nil

Conflict of interest: None

\section{INTRODUCTION}

Composite resins are the most commonly used direct tooth-colored restorations. Marginal integrity is one of

${ }^{1}$ Postgraduate Student, ${ }^{2}$ Associate Professor, ${ }^{3}$ Professor

${ }^{1-3}$ Department of Conservative Dentistry and Endodontics, SRM Dental College, Chennai, Tamil Nadu, India

Corresponding Author: Madhana Madhubala M, Associate Professor, Department of Conservative Dentistry and Endodontics, SRM Dental College, Chennai, Tamil Nadu, India, e-mail: dentistmadhana@yahoo.com the factors that decide the longevity of these restorations. Even though the marginal seal is achieved by micromechanical adhesion through etching and bonding procedures, secondary caries at the resin-tooth interface continues to be the most common failure of composite restorations. $^{1}$

Also, polymerization shrinkage and resultant contraction gaps can be associated with the encroachment of cariogenic bacteria at the resin-dentin interface from the saliva and smear layer which also accounts for the occurrence of secondary caries. ${ }^{2}$ Even a perfect resindentin interface can fail over a period due to hydrolytic and proteolytic degradation of the hybrid layer. ${ }^{3}$ Recent research focuses on the development of dental adhesives with antibacterial, water compatible and esterase resistant ability along the resin-dentin interface to enhance both the biological and mechanical integrity of resin-dentin bonding.

The acid component of etch and rinse adhesives with low $\mathrm{pH}$ have demonstrated antibacterial activity. ${ }^{4}$ However, certain bacteria are acid-resistant, and the buffering capacity of dentin can neutralize the effects of the acid. ${ }^{5}$ Various studies have been reported on the inability of total etch adhesives to inhibit bacterial growth or secondary caries. ${ }^{6}$

Literature shows various attempts at incorporation of antibacterial components such as fluorides, antibiotics methacryloyloxy dodecyl pyridinium bromide (MDPB), dimethylamino dodecyl methacrylate in dentin adhesives. ${ }^{7-9}$ Tziafas et al. reported that MDBP containing adhesives maintained pulp vitality but interfered with reparative dentin formation in exposed, infected pulp. ${ }^{10}$ In addition, these resins do not affect biofilm formation or initial adherence of $S$. mutans. ${ }^{11-13}$ Thus, there is a need for a unique adhesive to provide long-lasting antibacterial activity, increased bond strength, and durability.

Dopamine (DOPA) is a synthetic mimetic of mussel adhesive protein. Polydopamine is spontaneously formed by the oxidative polymerization of dopamine in aqueous solutions. ${ }^{14}$ It is a small molecule compound that contains both 3, 4-dihydroxy-L-phenylalanine and amine. It shows a good adhesive property to various organic and inorganic substrates under wet conditions. ${ }^{15}$ The adhesive property of mussels to the underwater surface is mostly due to the exhaustively repeated units of DOPA 
and amine. ${ }^{16}$ Nowadays, it is commonly used as surface modifiers to most of the medical appliance. Catecholfunctionalized synthetic polymer of dopamine has been showed to increase the bond strength between dentin and resin material. ${ }^{17}$ It also exhibits potent antibacterial activity against most common oral pathogens. Polydopamine Induced-polyethylene glycol coating proved to control and inhibit the cariogenic plaque formation on root surface. ${ }^{18}$

Both the adhisive and antibaterial characteristic of DOPA had lead to the new concept of incorporating this as a potent ingredient to the dental adhesive system. As S. mutans plays a vital role in the initiation and progression of caries, this study aimed to preliminarily evaluate the antibacterial effect of polydopamine incorporated totaletch adhesive system against $S$. mutans.

\section{MATERIALS AND METHODS}

\section{Preparation of Experimental Adhesive System}

Dopamine (DOPA) powder (Sigma Aldrich H8502,10 G) of $2 \mathrm{mg}$ was added in Tris Buffer solution $(1 \mathrm{mM})$ to 1 $\mathrm{mL}$ to form polydopamine. The optimal percentage of dopamine to be added to etch and rinse was determined by a pilot study of using 1, 3, 5 and $10 \% \mathrm{wt} \%$ of dopamine incorporated bonding agent (BA). We evaluated the degree of conversion using Fourier transform infrared spectroscopy and found that addition of upto 3 and $5 \mathrm{wt} \%$ of dopamine did not have any effect on polymerization. Hence, $3 \mathrm{wt} \%$ was selected to be added to fifth-generation bonding agent (total etch-Te-Econom Bond, Ivoclar Vivadent) vial of $2 \mathrm{~mL}$ to obtain a mixture of $3 \mathrm{wt} \%$. The vial was kept in a cyclomixer (Sigma scientific instruments, India) for 1 minute to obtain a uniform mix.

\section{Inoculation of Streptococcus Mutants and Agar Diffusion Test}

Round wells of around $6 \mathrm{~mm}$ diameter were created in the center of the agar plates by using a sterile conventional punch. Inoculation was performed by dipping a sterile swab in the S. mutans broth which was standardized according to $0.5 \mathrm{McFarland}$ standard and then brushing across the culture media in a zigzag fashion starting from the left top end and ending at the right bottom end. Then turning the plates clockwise and repeating the same so that the inoculation is uniform throughout the culture plate.

The experimental groups consisted of groups as follows: group I (DOPA 3\%), group II (DOPA 3\% + Bonding agent), group III (Bonding agent) and group IV (no material). For each group, 12 plates were used to evaluate the efficacy. Specimens for each group were prepared by light curing $1 \mathrm{~mL}$ of the bioactive ${ }^{6}(\mathrm{BA})$ in a dispensing well for 20 seconds, the created wells of each culture plate were filled completely with the experimental cured adhesives. Then, the inoculated agar plates were incubated aerobically for 24 hours at $37^{\circ} \mathrm{C}$.

\section{Measurement of the Zone of Inhibition}

After 24 hours of incubation at $37^{\circ} \mathrm{C}$, the inoculated agar culture plates were analyzed for the zone of inhibition under proper illumination. The zone of inhibition was seen as a round to oval clear area around the central well devoid of any bacterial growth. For measuring the zone of inhibition for each culture plate, the shortest diameter of the inhibition zone was measured as D1, and the longest diameter was measured as D2, and the average of the two was recorded as the "diameter of the zone of bacterial inhibition." The measurements were charted in Table 1 for each group and statistically analyzed using the Kruskal-Wallis test. Mann-Whitney U test was used for pairwise comparison.

\section{RESULTS}

All the groups resulted in a statistically significant difference in the zone of bacterial inhibition. Negative control showed no zone of inhibition. Based on the mean diameters, group II (Fig 1) showed the maximum zone of inhibition, and it exhibited statistically higher antibacterial activity than group III and group $1(\mathrm{p}<0.01)$.

\section{DISCUSSION}

The recent development of adhesives is evolving with antibacterial additives. An antibacterial adhesive should inhibit the biofilm accumulation on the material surface and the restoration interface to prevent the detrimental effects such as pulp damage, hypersensitivity, and recurrent

Table 1: Comparison of mean diameter (zone of inhibition) by various groups

\begin{tabular}{lll}
\hline Groups & Mean Value (SD) & $p$-value \\
\hline Group & $11 \mathrm{~mm}(1.44)$ & $\mathrm{p}<0.01$ \\
Group II & $21 \mathrm{~mm}(1.03)$ & \\
Group III & $9 \mathrm{~mm}(2.03)$ & \\
Group IV & $0 \mathrm{~mm}(0)$ & \\
\hline
\end{tabular}

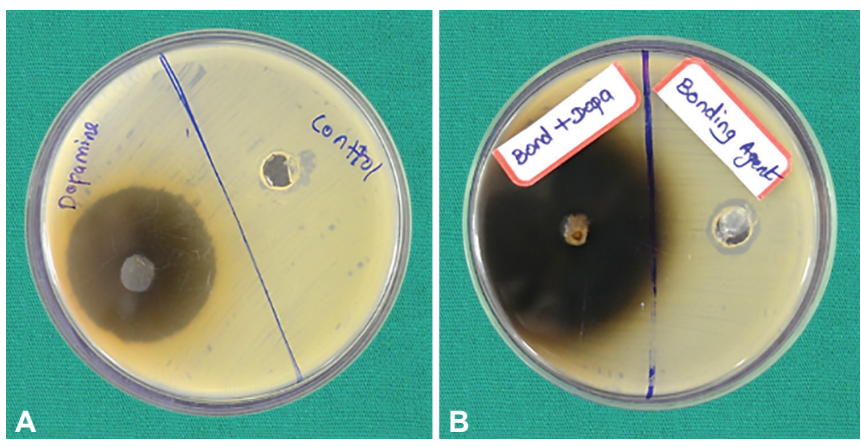

Figs 1A and B: Zones of Inhibition of various groups 
caries resulting from residual bacteria or microleakage. Thus, enhanced antibacterial action at the tooth-restoration interface will be highly beneficial. A tight biologic seal can improve the longevity of the restoration.

S. mutans was selected in this study for evaluating the antibacterial activity as it is one of the main causative pathogens for dental caries. S. mutans can metabolize carbohydrates to acids, leading to demineralization of the tooth structure and the tooth-restoration margins. Also, the bacterium can synthesize extracellular polysaccharides. ${ }^{19,20}$ Therefore an antibacterial adhesive system against $S$. mutans could be a promising approach to prevent recurrent caries.

Although the antibacterial activity of adhesive materials is an important factor in terms of reduction of secondary caries, studies have shown that the incorporation of antibacterial agents could impair mechanical properties. Moreover, the release of the antibacterial substance from the material could result in further changes in physical properties of adhesive interface. ${ }^{21}$

The present study is first of its kind on the incorporation of antibacterial wet adhesive DOPA component in the dental adhesive system. The chemical structure of polydopamine lies in its ability to incorporate many functional groups such as catechol, amine, and imine. These functional groups can serve as attachment anchors with desired molecules. ${ }^{22}$ Lee et al. evaluated the potential of catechol-functionalized modified polymer with $\mathrm{Fe}^{3+}$ additive improved the bond strength of commercial adhesive resin to the saliva contaminated dentin surface. ${ }^{17}$

The results of our study suggest that the total-etch used alone showed markedly low antibacterial effect than polydopamine incorporated. The $\mathrm{pH}$ value of Te-Econom (adhisive system) bond is very low ( $\mathrm{pH} 1.7)$, and this can be attributed to the minimal inhibitory effects against the bacteria used in our study. In another study, a totaletch adhesive system with $\mathrm{pH} 2.5$ showed no inhibition zones against $S$. mutans and L. casei. ${ }^{23}$ This study result corroborates with the antibacterial activity of adhesive system reported by Atac et al. ${ }^{24}$ and Baseren et al. ${ }^{25}$

The DOPA itself has intrinsic antimicrobial activity. ${ }^{26}$ This could be the reason for the results of group I which showed $11 \mathrm{~mm}$ of the zone of inhibition. Maji et al. showed that antimicrobial activity of various commonly used antibiotics is enhanced against most of the virulent bacteria when combined with dopamine hydrochloride (HCL). ${ }^{27}$ The presence of benzene ring in dopamine may be responsible for the antimicrobial activity. The mode of action of dopamine on gram positive and negative bacteria could restrict the growth of bacterial cells and prevent their further multiplication. Bacteria could be encapsulated with a thickness of $120 \mathrm{~nm}$ polydopamine shell as indicated by UV and fluorescence spectroscopy. ${ }^{26}$
The DOPA incorporated total-etch adhesive system showed statistically higher antibacterial activity than other groups. Thus, the DOPA-functionalized total-etch adhesive showed synergistic activity when compared to other groups. This could also be due to the local toxic effects of active groups such as phenolic hydroxyl/ o-quinone and amino/imino on the outer membrane of bacterial cells by reducing the permeability to specific components that are necessary to bacterial cell survival or preventing metabolic waste from releasing. ${ }^{28}$ Mei et al. proved that polydopamine-induced-polyethylene glycol could prevent cariogenic biofilm formation in the form of mouth rinse. He also suggested that it could be an additive to varnish to prevent biofilm adhesion on the tooth. $^{18}$

As studies have proven that DOPA enhances wet adhesion also ${ }^{29}$ DOPA incorporated total-etch adhesive system can be a promising antibacterial additive to dental adhesive systems to improve both biological seal and bond strength at the resin-dentin interface.

\section{CONCLUSION}

Within the limitations of this study, dopamine incorporated total-etch adhesive system exhibited significantly higher antibacterial action against S. mutans. Further, in vivo studies are needed to prove the antibacterial effect of this novel adhesive systems to increase the longevity of dental restorations.

\section{CLINICAL SIGNIFICANCE}

Dopamine (DOPA) can be a promising antibacterial additive to dental adhesive systems to improve both biological seal and bond strength at the resin-dentin interface.

\section{REFERENCES}

1. Chrysanthakopoulos NA. Reasons for Placement and Replacement of Resin-based Composite Restorations in Greece. J Dent Res Dent Clin Dent Prospects. 2011;5(3):87-93.

2. Soares CJ, Faria ES, Rodrigues MDP, Vilela ABF, Pfeifer CS, Tanbiio DM et al. Polymerization shrinkage stress of composite resins and resin cements What do we need to know? Braz. Oral Res. 2017;31(suppl):e62:49-63.

3. Liu Y, Tjäderhane L, Breschi L, Mazzoni A, Li N, Mao J, Tay FR. Limitations in Bonding to Dentin and Experimental Strategies to Prevent Bond Degradation. J Dent Res. 2011;90:953-968.

4. Amin S, Shetty HK, Varma RK, Amin V, Nair PMS. Comparative evaluation of antibacterial activity of total-etch and self-etch adhesive systems: An ex vitro study. J Conserv Dent. 2014;17(3):266-270.

5. Camps J, Pashley DH. Buffering action of human dentin in vitro. J Adhes Dent 2000;2(1):39-50.

6. Bapna MS, Murphy R, Mukherjee S. Inhibition of bacterial colonization by antimicrobial agents incorporated into dental resins. J Oral Rehab. 1988;15:405-411. 
7. Cheng L, Zhang K, Melo MA, Weir MD, Zhou X, Xu HH. Antibiofilm dentin primer with quaternary ammonium and silver nanoparticles. J Dent Res. 2012;91:598-604.

8. Imazato S, Kuramoto A, Takahashi Y, Ebisu S, Peters MC. In vitro antibacterial effects of the dentin primer of Clearfil Protect Bond. Dent Mater. 2006;22:527-532.

9. Cheng L, Weir MD, Xu HH, Kraigsley AM, Lin NJ, Lin GS, Zhou X. Antibacterial and physical properties of calciumphosphate and calcium-fluoride nanocomposites with chlorhexidine. Dent Mater. 2012;28:573-583.

10. Tziafas D, Kolinioto-K E, Tziafa C, Papadimitriou S. Effects of a new antibacterial adhesive on the repair capacity of the pulpdentine complex in infected teeth. Int Endod J. 2007;40(1): 58-66.

11. Lobo MM, Goncalves RB, Pimenta LA, Bedran-Russo AK, Pereira PN. In vitro evaluation of caries inhibition promoted by self-etching adhesive systems containing antibacterial agents. J Biomed Mater Res B Appl Biomater. 2005;75(1):122-127.

12. Rolland SL, McCabe JF, Robinson C, Walls AW. In vitro biofilm formation on the surface of resin-based dentine adhesives. Eur J Oral Sci. 2006;114(3):243-249.

13. Hahnel S, Leyer A, Rosentritt M, Handel G, Burgers R. Surface properties and in vitro Streptococcus mutans adhesion to selfetching adhesives. J Adhes Dent. 2009;11(4):263-269.

14. Ding YH, Floren M, Tan W. Mussel-inspired polydopamine for bio-surface functionalization. Biosurface and Biotribology. 2016;2(4):121-136.

15. Jo SH, Sohn JS. Biomimetic Adhesive Materials Containing Cyanoacryl Group for Medical Application. Molecules. 2014;19(10): 1679-1693.

16. Ho CC, Ding SJ. Structure, properties, and applications of mussel-inspired polydopamine. J Biomed Nanotechnol. 2014;10(10):3063-3084.

17. Sang BL, Carlos GC, Kwang MK, Kyoung NK, Kenichi K. Catechol-Functionalized Synthetic Polymer as a Dental
Adhesive to Contaminated Dentin Surface for a Composite Restoration. Biomacromolecules. 2015;16(8):2265-2275.

18. Mei ML, Li QL, Chu CH. Inhibition of cariogenic plaque formation on root surface with polydopamine-induced-polyethylene glycol coating. Materials (Basel). 2016;9(6):414-426.

19. Harper DS, Loesche WJ. Growth and acid tolerance of human dental plaque bacteria. Arch Oral Biol 1984;;29(10):843-848.

20. Kuramitsu HK. Virulence factors of mutans streptococci role of molecular genetics. Crit Rev Oral Biol Med. 1993;4(2):159-176.

21. Vanajasan PP, Dhakshinamoorthy M, Rao CS. Factors affecting the bond strength of self-etch adhesives: A meta-analysis of literature. J Cons Dent 2011;14(1):62-67.

22. Dalsin JL, Hu BH, Lee BP, Messersmith PB. Mussel adhesive protein mimetic polymers for the preparation of nonfouling surfaces. J Am Chem Soc 2003;125(14):4253-4258.

23. Cristiane D, Thais CN, Denise MPS. Josimeri H. Effect of light-activation on the antibacterial activity of dentin bonding agents 2009;8(4):175-180.

24. Atac AS, Cehreli ZC, Sener B. Antibacterial activity of fifth-generation dentin bonding systems. J Endod. 2001;27(12):730-733.

25. Baseren M, Yazici AR, Ozalp M, Dayangac B. Antibacterial activity of different generation dentin-bonding system. Quintessence Int. 2005;36(5):339-344.

26. Iqbal Z, Lai EPC, Avis TJ. Antimicrobial effect of polydopamine coating on Escherichia coli. J Mater Chem 2012;22(40): 21608-21612

27. Maji S, Maji HS, Chakraborthy P, Dastidar S. potential of dopamine hydrochloride as an novel antimicrobial agent. International journal of biomedical and pharmaceutical sciences. 2010;4(2):70-75.

28. Ji Y, Ji T, Liang K, Zhu L. Mussel-inspired soft-tissue adhesive based on poly(diol citrate) with catechol functionality. J Mater Sci Mater Med 2016;27(2):30.

29. Yang FK, Zhao B. Adhesion properties of self-polymerized dopamine thin film, Surface Science 2011;3(1):115-122. 